\title{
A VERY SLOWLY CONVERGENT SEQUENCE OF CONTINUOUS FUNCTIONS
}

\author{
WALTER RUDIN ${ }^{1}$
}

\begin{abstract}
A sequence of continuous functions $f_{n}:[0,1] \rightarrow(0,1]$ is constructed, with $\lim _{n \rightarrow \infty} f_{n}(x)=0$ for every $x \in[0,1]$, but such that to every unbounded sequence $\left\{\lambda_{n}\right\}$ of positive numbers corresponds a point $x \in[0,1]$ at which $\lim \sup _{n \rightarrow \infty} \lambda_{n} f_{n}(x)=\infty$.
\end{abstract}

This may be surprising since the sequence $\left\{f_{n}\right\}$ is completely determined by its values on a countable dense set, and since to every countable collection $\left\{S_{i}\right\}$ of numerical sequences that tend to 0 there corresponds a sequence $T$, with $T(n) \rightarrow \infty$, such that $\lim _{n \rightarrow \infty} T(n) S_{i}(n)=0$ for all $i$.

Each $x \in K$ (the Cantor set) has a unique representation

$$
x=\sum_{n=1}^{\infty} 3^{-n} a_{n}(x)
$$

where $a_{n}(x)$ is 0 or 2 . Define functions $g_{n} \in C(K)$ by

$$
\begin{array}{ll}
g_{n}(x)=a_{1}(x)+\cdots+a_{n}(x) & \text { if } a_{n}(x)=2, \\
g_{n}(x)=2 n-1 & \text { if } a_{n}(x)=0 .
\end{array}
$$

If $x \in K$ is fixed, then $\left\{g_{n}(x)\right\}$ is a sequence of positive integers in which none occurs twice; thus $g_{n}(x) \rightarrow \infty$ as $n \rightarrow \infty$. If $\delta_{n}>0$ and $\lim$ inf $\delta_{n}=0$, there exist integers $1<n_{1}<n_{2}<\cdots$ such that $r^{2} \delta_{n_{r}}<1 \quad(r=1,2,3, \cdots)$. Choose $x \in K$, corresponding to $\left\{\delta_{n}\right\}$, by specifying that $a_{n_{r}}(x)=2$ for all $r$, and that $a_{n}(x)=0$ otherwise. Then $\delta_{n_{r}} g_{n_{r}}(x)=2 r \delta_{n_{r}}<2 / r$ so that $\lim \inf \delta_{n} g_{n}(x)=0$.

To complete the construction, put $f_{n}(x)=1 / g_{n}(x)$ if $x \in K$, and define $f_{n}$ on the rest of $[0,1]$ by linear interpolation.

REMARK 1. These $f_{n}$ are piecewise linear. There are polynomials $P_{n}$ such that $f_{n}<P_{n}<2 f_{n}$. This yields a sequence of polynomials with the properties stated in the Abstract.

REMARK 2. On the other hand, if $X$ is compact, $f_{n}: X \rightarrow(0, \infty)$ is continuous, and $\lim f_{n}(x)=0$ for every $x \in X$, then there does exist $\left\{\lambda_{n}\right\}$ such

Received by the editors November 6,1972 and, in revised form, November 21, 1972. AMS (MOS) subject classifications (1970). Primary 40A05, 41A25.

${ }^{1}$ Partially supported by NSF Grant GP-24182. 
that $\lambda_{n} \rightarrow \infty$ and $\lim _{\inf _{n \rightarrow \infty}} \lambda_{n} f_{n}(x)=0$ for every $x \in X$. To see this, put

$$
h_{n}=\min \left(f_{1}, \cdots, f_{n}\right), \quad m_{n}=\max _{x} h_{n}(x), \quad \lambda_{n}=1 / \sqrt{ } m_{n} .
$$

Since $h_{n}(x) \rightarrow 0$ monotonically and $X$ is compact, $m_{n} \rightarrow 0$. To each $x \in X$ corresponds a sequence $\left\{n_{i}\right\}, n_{i} \rightarrow \infty$, such that $f_{n_{i}}(x)=h_{n_{i}}(x)$. For this $\left\{\boldsymbol{n}_{\boldsymbol{i}}\right\}$,

$$
\lambda_{n_{i}} f_{n_{i}}(x)=\lambda_{n_{i}} h_{n_{i}}(x) \leqq \lambda_{n_{i}} m_{n_{i}} \rightarrow 0 \quad \text { as } i \rightarrow \infty .
$$

Department of Mathematics, University of Wisconsin, Madison, Wisconsin 53706 